\title{
A tilapicultura e seus insumos, relações econômicas
}

\section{João Donato Scorvo Filho${ }^{1}$, Célia Maria Dória Frascá-Scorvo르, João Manoel Cordeiro Alves², Fernanda Rinaldi Alves de Souza ${ }^{3}$}

\footnotetext{
${ }^{1}$ Pesquisador do Pólo Apta do Leste Paulista - SAA-SP.

${ }^{2}$ Gerente de Produtos para Aquicultura da Guabi Nutrição Animal.

3 Oceanógrafa autônoma.
}

RESUMO - A aquicultura brasileira é a segunda (destaque para o Chile) em produção sul-americana, apoiada basicamente nas cadeias de produção da tilapicultura e da carcinicultura. O Brasil produziu, em 2007, 95.691,0 toneladas de tilápia, o que representa $45 \%$ da produção da aquicultura continental. A aquicultura formada por pequenos e médios produtores vem mostrando, nos últimos anos, mudança nos sistemas de criação. Até a o final da década de 90, ela se baseava no sistema semiintensivo em viveiros escavados e de barragens. A partir do ano de 2000, surge, com força, a tilapicultura em tanques-rede, principalmente em águas da União (grandes reservatórios de hidroelétricas e açudes da Região Nordeste). Esta mudança acarretou alterações na cadeia de produção, uma vez que são necessários insumos adequados ao sistema: rações específicas, material genético compatível com a criação e formas de escoamento da produção, uma vez que no novo sistema apresenta maior escala de produção. O sistema agroindustrial do pescado abrange dois sistemas de produção distintos: a pesca (sistema extrativo) e a aquicultura (sistema produtivo). Alguns elos deste sistema são compartilhados pelos dois sistemas, porém ração e material genético são exclusivos da aquicultura. Nos elos de transformação, distribuição e comercialização, ambos os sistemas se relacionam e, muitas vezes, concorrem entre si. Atualmente, o Brasil possui linhagens melhoradas geneticamente, em contínuo processo de melhoramento e adaptaçao às nossas condições. A atividade de tilapicultura vem passando, desde as décadas passadas, por um processo de profissionalização, com o produtor mais atento ao manejo e aos insumos utilizados. Hoje, é possível escolher o insumo que mais se adapta ao manejo empregado pelo produtor, porém isso não é é uma prática generalizada quando se considera seu preço de venda.

Palavras-chave: alevinos, insumos, ração, tilápia

\section{Tilapia culture and its inputs, economic relations}

ABSTRACT - The Brazilian aquaculture is the second (Chile is the first one) in South American production, supported basically by production chains of shrimp and tilapia culture. Brazil produced, in 2007, 95691.0 tons of tilapia, representing $45 \%$ of the continental aquaculture. Aquaculture from small and medium producers has shown in recent years changes on farming systems. By the end of the 90s, it was based on semi-intensive ponds and dams. From the year 2000, tilapia culture in cages highlighted, especially in Union waters (large reservoirs and hydroelectric dams in the Northeast Region). This change led to changes in the production chain, because adequate inputs to the system is needed: special diets, genetic material compatible with the rearing phases and production flow, since the new system has a higher scale of production. The agro-industrial system of fish covers two distinct production systems: a fishery system (extraction) and aquaculture (production system). Some links in this system are shared by both systems, but food and genetic material are exclusive of aquaculture. Both systems interact with processing, distribution and marketing, and often compete with each other. Currently, Brazil has genetically improved strains, continuous process improvement and adaptation to our conditions. The activity of tilapia culture has increased through a process of professionalization, where the producer is closer to the used management and inputs. Today, producers can choose the input that best fits the management practices, but this is not a common practice when considering price.

Key Words: inputs, fingerlings, ration, tilapia 


\section{Introdução}

\section{O agronegócio mundial}

A revista britânica The Economist, publicou em agosto de 2003, uma reportagem especial, como matéria de capa: Piscicultura, a promessa de uma revolução azul (Fish farming, the promise of a blue revolution). A matéria foi muito importante para a aquicultura, principalmente pela abrangência mundial da revista, levantando as atenções para a atividade. A matéria inicia com um subtítulo: Como a aquicultura pode atender a demanda mundial por peixes sem degradar o meio ambiente (How aquaculture might meet most of the world's demand for fish without ruining the enviroment) e coloca com primeira frase do texto: A piscicultura tem má reputação (Fish farming has a bad reputation). Apesar do impacto negativo desta introdução, durante o texto, a critica é amenizada e mostra que o pescado é um produto importante que tem sua procura aumentada e que, a aquicultura é a única solução para abastecer o mercado. Faz um comparativo entre a revolução verde, ocorrida na agricultura (terra), e a revolução azul, que acontecerá com a aquicultura (água). Esta comparação levanta a discussão sob os métodos empregados pela agricultura e, que precisam ser repensados pela aquicultura (uso de fertilizantes, alta mecanização, biocidas, etc.).

A lembrança deste artigo, quase sete anos depois, é proposital, pois levanta a discussão do que vem acontecendo na aquicultura no mundo.

Segundo a FAO (2009), o pescado tem se globalizado e cerca de $37 \%$ de toda a produção pesqueira (53 milhões de toneladas) se comercializa em nível internacional. Em 2008 as exportações de pescados geraram 102.000 milhões de dólares. Outra informação importante é que $60 \%$ do comercializado se origina em países em desenvolvimento, representado $80 \%$ do valor monetário. Os países desenvolvidos (Europa, Japão e Estados Unidos) são responsáveis pela importação de $70 \%$ do pescado comercializado.

É neste contexto que o Brasil se insere como grande fornecedor de pescado para o mundo. Com potencial hídrico e ambiental e com possibilidades de não cometer os mesmos erros que outros já cometeram, comprometendo o ambiente onde se produziam seus pescados.

Especificamente sobre a criação de tilápias, ou tilapicultura, Fitzsimmons (2010) relata um crescimento mundial da produção de tilápia de aproximadamente 2,8 milhões de toneladas em 2008 e deve aumentar para 3,0 milhões de toneladas em 2010. Nos EUA e na China, a produção diminuiu ligeiramente em 2008, na China devido ao rigoroso inverno e nos EUA devido à dura recessão. O mesmo autor informa que a produção mundial da tilápia vem aumentando pela popularização da tilápia nos EUA e na União Européia e China, continua sendo ainda o maior produtor mundial.

O Brasil hoje produziu, em 2007, 95.691,0 toneladas de tilápia representando $45 \%$ da produção da aquicultura continental, produção esta que mostra que a tilapicultura no Brasil tem uma contribuição importante para o crescimento da aquicultura nacional.

\section{Estrutura da cadeia de produção da aquicultura}

É inegável a importância para a aquicultura a criação da Secretaria Especial de Aquicultura e Pesca (SEAP/PR), transformada em Ministério da Pesca e Aquicultura (MPA) em junho de 2009. Depois da extinção da Superintendência do Desenvolvimento da Pesca (SUDEPE) em 1989, tanto a pesca como a aquicultura ficaram órfãos de uma entidade fomentadora, sendo consideradas pelo órgão ambiental Instituto Brasileiro do Meio Ambiente e dos Recursos Naturais Renováveis - IBAMA - como impactantes e não merecedoras de incentivos ou fomentos.

A aquicultura nacional baseia-se essencialmente nas cadeias de produção da tilápia e do camarão marinho (vannamei), o que leva a estar como o segundo país sulamericano em produção, sendo o Chile o maior produtor (Tabela 1).

A aquicultura formada por pequenos e médios produtores vem mostrando, nos últimos anos, mudança nos sistemas de criação. Até a o final da década de 90 ela se baseava no sistema semi-intensivo em viveiros escavados e de barragens. A partir do ano de 2000 surge, com força, a tilapicultura em tanques-rede, principalmente em águas da União (grandes reservatórios de hidroelétricas) mudança

Tabela 1 - Produção da aquicultura na América do Sul e participação percentual por país, no ano de 2006

\begin{tabular}{lcc}
\hline Pais & Produção $(\mathrm{t})$ & $\%$ \\
\hline Chile & 802.410 & 63,218 \\
Brasil & 271.896 & 21,421 \\
Equador & 78.300 & 6,169 \\
Colômbia & 60.100 & 4,735 \\
Peru & 28.393 & 2,237 \\
Venezuela & 22.210 & 1,750 \\
Argentina & 2.528 & 0,199 \\
Paraguai & 2.100 & 0,165 \\
Guiana & 660 & 0,052 \\
Bolívia & 430 & 0,034 \\
Suriname & 180 & 0,014 \\
Guiana Francesa & 37 & 0,003 \\
Uruguai & 37 & 0,003 \\
Total & 1.269 .281 & 100,00 \\
\hline
\end{tabular}

Fonte: ftp://ftp.fao.org/fi/stat/summary/summ_06/a-0a.pdf 
esta, que trouxe alterações na cadeia de produção, uma vez que, são necessários insumos adequados ao sistema, como rações específicas, material genético compatível com a criação e mesmo formas de escoar a produção, uma vez que no novo sistema apresenta maior escala de produção.

A produção brasileira em tanques-rede é baseada, quase que $100 \%$, na criação de tilápias.

Cabe aqui fazer um parêntese e comentar sobre a carcinicultura, que também, vem apresentando mudanças em seu sistema de criação tendo sido afetada por doenças e restrições de mercado. A criação de camarão tem se adaptado a novas situações, com uso de menor densidade de estocagem nos viveiros, manejos sanitários e profiláticos e produtos mais adequados ao mercado interno.

Importante destacar que em 2003 foram produzidas 90.190 toneladas de camarão cultivado sendo exportadas 58.455 toneladas (64.8\%). Está produção reduziu para 70.0000 toneladas em 2008 (queda de 22,4\%) quando foram exportadas 9.397 toneladas (13,4\%), mostrando aumento de 91\% no consumo interno (Rocha \& Rocha, 2008).

As demais atividades relacionadas com a aquicultura, como a malacocultura, ranicultura e algicultura, mesmo importantes, apresentam menor volume de produção e valor econômico.
Relações econômicas dos insumos da aquicultura

O sistema agroindustrial do pescado abrange dois sistemas de produção distintos, a pesca, sistema extrativo, e a aquicultura, sistema produtivo (Figura 1).

Alguns elos deste sistema são compartilhados pelos dois sistemas, sendo os insumos ração e material genético, exclusivos da aquicultura.

Nos elos de transformação, distribuição e comercialização os dois sistemas se relacionam, em muitas das vezes até concorrendo entre si.

\section{Material genético}

A piscicultura até meados da década de 80 , tinha como foco a produção de alevinos de espécies exóticas (carpa comum, tilápias, black bass, etc.) para a distribuição gratuita ou venda, existindo poucos piscicultores que produziam peixes para o consumo. Este foco era o mesmo para as instituições governamentais e comerciais. Os proprietários rurais, que possuíam lagos e represas em suas terras, procuravam produtores e órgãos governamentais, para obterem formas jovens e, assim povoar seus corpos d'água. As concessionárias dos reservatórios de hidroelétricas e instituições de ensino, pesquisa e fomento foram grandes distribuidores de alevinos e importantes no fomento da piscicultura.

\section{Sistema Agroindustrial (SAG) do Pescadc}

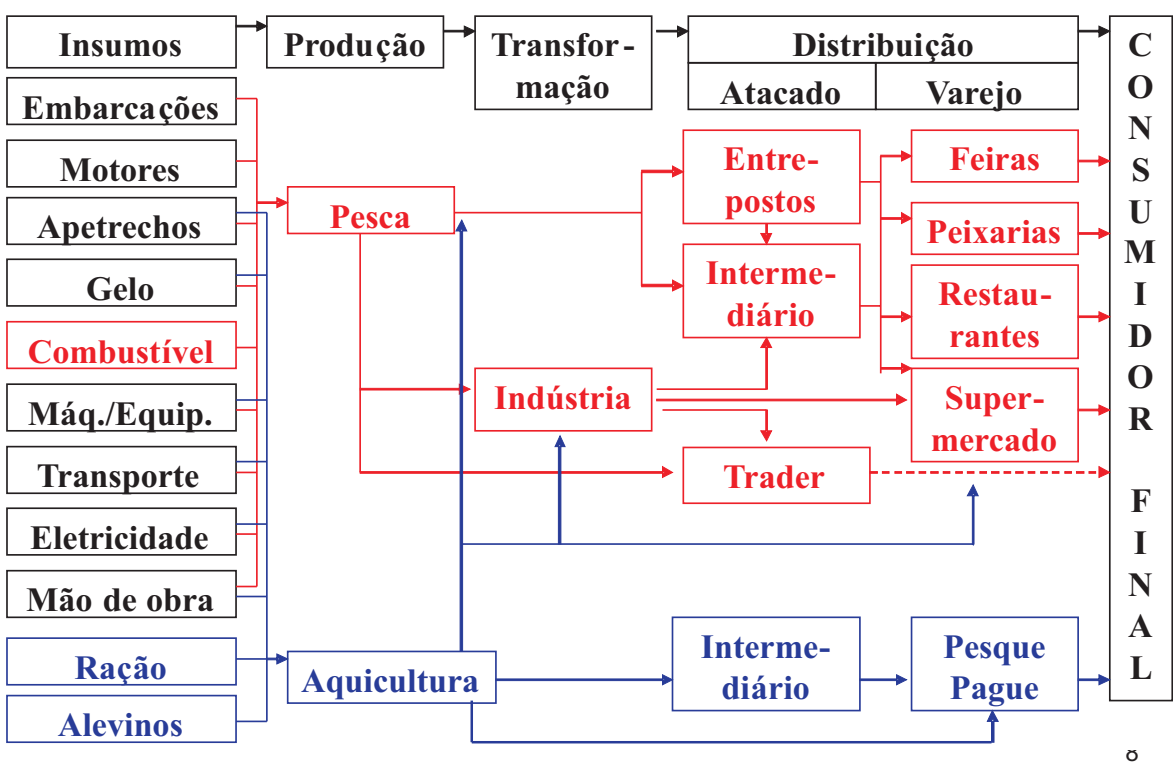

Fonte: Sonoda, 2010 - comunicação pessoal.

Figura 1 - Sistema Agroindustrial do Pescado. 
Na década de 90 com o aumento da produção houve a necessidade de mais formas jovens para atender a demanda de pós-larvas e alevinos.

Segundo Zimmermann (2002), a tilápia foi introduzida na América Latina no final da década de 40 e no início da década de 50. As primeiras espécies de tilápias provavelmente mossambica, rendalli e zilli, foram introduzidas no Brasil e outros paises como, Jamaica, República Dominicana, Trinidad e Tobago, Ilhas Virgens e Guiana. No final dos anos 50, ela foi introduzida Porto Rico, El Salvador, Colômbia, Venezuela, Equador, Guatemala e Honduras, enquanto no México, Cuba e Peru a sua introdução ocorreu na década de 60 . Somente mais tarde, ou seja, na década de 70 , é que ela foi introduzida no Uruguai, Argentina e Bolívia. Atualmente, estão sendo cultivadas em toda a América Latina.

A introdução da tilápia-do-nilo ou nilótica (Oreochromis niloticus), na década de 70, em alguns paises da América Latina, começa a mudar o cenário da tilapicultura neste continente, pois, ainda, segundo Zimermann (2002) esta espécie apresenta excelente desempenho zootécnico em relação às outras espécies introduzidas anteriormente.

O Brasil hoje, já possui algumas linhagens melhoradas geneticamente e que continuam sendo melhoradas e adaptadas as nossas condições que são elas:

- Tilápia Tailandesa ou Chitralada: que chegou ao Brasil em 1996, doação do Asian Institute of Technology (AIT), e que, segundo Santos (2006), foi desenvolvida no Japão e melhorada no Palácio Real Chitralada na Tailândia em 1965. O melhoramento genético feito pela empresa GENOMAR, produziu a tilápia chamada de SUPREME TILÁPIA, nome mundialmente conhecido e registrado, no Brasil se chama SUPREME TILÁPIA AQUABEL.

- Linhagem GIFT (Genetic Improved Farmed Tilapia): Em março de 2005, a Estação Experimental da Universidade Estadual de Maringá (UEM - Codapar) recebeu tilápias, representantes de 30 famílias, da linhagem GIFT, a partir de um projeto, apoiado pela Secretaria Especial de Aquicultura e Pesca - SEAP/PR, elaborado em conjunto com o World Fish Center. Essa linhagem é fruto de um melhoramento genético executado nas Filipinas a partir de uma ampla base genética de linhagens selvagens e de criação.

- GMT(Genetically Male Tilápia): a linhagem GMT® desenvolvida pela empresa Fishgen, que aplicou uma técnica de manipulação genética para produção total de machos, conhecida como "tecnologia YY", que consiste num programa de criação combinando a feminização e seleção de alevinos, para promover machos com genótipo YY, ao invés do usual XY do genótipo macho. Os machos YY são conhecidos como "Supermachos" e tem a propriedade de conceber somente alevinos machos. Esta prole é denominada “Genetically Male Tilapia” (GMT®) e são machos absolutamente normais. O GMT® foi desenvolvido primeiramente nas Filipinas e a Fishgen, junto com seus associados, é a única empresa capaz de produzir o Supermacho YY. No Brasil, esta tecnologia está sendo representada pela Fazenda Santa Isabel sediada no Estado de São Paulo. Esta linhagem tem duas variedades:

- Tilápia Prateada GMT®: proveniente de variedades selecionadas na África do Sul. Para atender às exigências do mercado de tilápia, a Fishgen e a Santa Isabel estão investindo em pesquisa de desenvolvimento e melhoramento de produtos.

- Tilápia Vermelha GMT®: proveniente de uma raça pura de $O$. niloticus vermelha.

Os dados apresentados por Valente (2000) mostram que, em 1998, haviam 237 laboratórios instalados no Brasil, com capacidade de produzir 798 milhões de alevinos (Tabela 2). A produção destes laboratórios era de, aproximadamente 450 milhões de alevinos. Segundo o Ibama (2000) o Brasil produziu, em 1998, 88.565,5 toneladas de pescado pela aquicultura continental o que corresponde a 190 gramas de peixe produzidos para cada alevino.

Castagnolli et al. (1996) mostram que a relação alevino - quilo de peixe produzido, em 1993, era de 4.978 gramas de pescado para cada alevino - 164 milhões de alevinos para uma produção de 33.042,7 toneladas de peixes.

Segundo Suplicy (2007), o Brasil produziu em 2005, em 175 laboratórios, 617,5 milhões de alevinos. Deste total, os alevinos de tilápia representaram 304,5 milhões (49,3\%), alevinos de tambaqui foram produzidos 52 milhões $(8,4 \%)$. Os 42,3\% restantes (261 milhões) representaram alevinos de 35 espécies de peixes diferentes. Ressalta a informação de Suplicy (2007) que desta produção, 33\% foi produzida na

Tabela 2 - Capacidade instalada e produção brasileira de alevinos por região (em milheiros)

\begin{tabular}{lccc}
\hline Região & \multicolumn{2}{c}{ Capacidade instalada } & \multirow{2}{*}{ Produção } \\
\cline { 2 - 3 } & Alevinos & Laboratórios* & \\
\hline Norte & 35.300 & 19 & 13.000 \\
Nordeste & 233.550 & 39 & 99.250 \\
Centro-oeste & 65.900 & 20 & 17.902 \\
Sudeste & 13.900 & 73 & 13.900 \\
Sul & 449.083 & 86 & 306.396 \\
Total & 798.083 & 237 & 450.448 \\
\hline
\end{tabular}

* Não foram informados o número de laboratórios nos estados de Minas Gerais, Rio de Janeiro, Paraná

Fonte: Valente et al. (2000). 
Região Nordeste do Brasil, ficando a Região Sudeste com $25 \%$, Sul com 22\%, Centro-oeste com 14 e $6 \%$ da Região Norte.

A relação, entre estas informações de produção de alevinos fornecidas por Suplicy (2007) com as informações de produção, apresentadas pelo IBAMA (2007) podem ser visualizadas na Tabela 3.

As relações apresentadas na Tabela 3 (média de $0,3 \mathrm{~kg}$ para cada alevino) estão mais próximas da relação conseguida pelos dados indicados por Valenti et al. (2000), (0,19 kg para cada alevino) do que as obtidas com os dados de Castagnolli et al. (1996) (4,98 kg para um alevino).

As informações atualizadas, para número de laboratórios e produção de alevinos não estão disponíveis. A publicação dos dados obtidos com o Censo Aquícola, que vem sendo realizado pelo Ministério da Pesca e Aquicultura, poderão trazer a luz, estas tão importantes informações.

Em relação à carcinicultura, o Brasil produziu em 2008, 70.000 toneladas.

$\mathrm{Na}$ análise dos custos de produção da piscicultura, relatados por Martin et al. (1995), podemos notar que o item alevino pode representar de 2,5 a 9,32\% da composição do

Tabela 3 - Produção de alevinos, em milhões de indivíduos, de peixes, em toneladas e relação entre o número de alevinos de indivíduos e de pescado produzidos ( $\mathrm{kg} \mathrm{x}$ alevinos), em 2005

\begin{tabular}{lccc}
\hline Região & Alevinos* & Pescado** & Relação Pesc. x Alev. \\
\hline Norte & 37,1 & 19675,5 & $530,3 \mathrm{~g}$ \\
Nordeste & 203,6 & 35228,0 & $173,0 \mathrm{~g}$ \\
Centro-oeste & 86,5 & 33303,5 & $385,0 \mathrm{~g}$ \\
Sudeste & 154,4 & 31335,0 & $202,9 \mathrm{~g}$ \\
Sul & 135,9 & 59204,5 & $435,6 \mathrm{~g}$ \\
Total & 617,5 & 178746,5 & $289,5 \mathrm{~g}$ \\
\hline
\end{tabular}

* Fonte: Suplicy (2007).

**Fonte: Ibama (2005). custo de produção da criação de tilápias, peixes redondos, carpas e bagre africano. O trabalho destes autores não levou em consideração a piscicultura em tanques-rede, estudando apenas o sistema semi-intensivo em viveiros escavados e de barragens.

Ao longo do tempo com a maior importância da piscicultura, outros trabalhos foram publicados mostrando que o material genético não pode ser menosprezado na análise da viabilidade econômica da atividade. A análise econômica da pratica da piscicultura intensiva em tanquesrede mostra que este item é mais representativo, como mostram os trabalhos de Carneiro et al. (1999), onde o item alevinos correspondeu a $17,11 \%$ do custo operacional efetivo, o trabalho de Campos et al. (2007), com uma representação no custo operacional efetivo de $13,54 \%$ para o item alevinos.

As informações sobre a série histórica dos preços pagos pelos produtores pelo material genético são raras. Scorvo Filho et al. (1999) apresentam os preços praticados no Estado de São Paulo, nos anos de 1995 e 1997. O preço médio do milheiro alevinos de 14 espécies no ano de 1995 foi de $\mathrm{R} \$ 104,08$ (o milheiro de menor valor foi o da tilápia com $\mathrm{R} \$ 60,00$ e o de maior valor o do black bass com $\mathrm{R} \$ 340,00$ ) e para o ano de 1997, com um aumento de 7,43\% esta média era de R \$111,82 (também tilápia e black bass com R \$66,99 - aumento de 11,65\% e R $\$ 380,00$ - aumento de 11,76\%).

Firetti \& Sales (2007) apresentam dados de valores praticados para a tilápia no Estado de São Paulo, para: alevinos, ração, peixe para venda, custo operacional efetivo médio e margem bruta média.

A partir destes valores foi elaborado o Figura 3, que demonstra a variação do preço do milheiro de alevino entre os anos de 1996 e 2006.

A análise feita por Firetti \& Sales (2007) mostra que o preço dos alevinos de tilápia reduziram 26,42\%. O valor do milheiro, que era praticado a $\mathrm{R} \$ 126,11$, em 1996 passou para

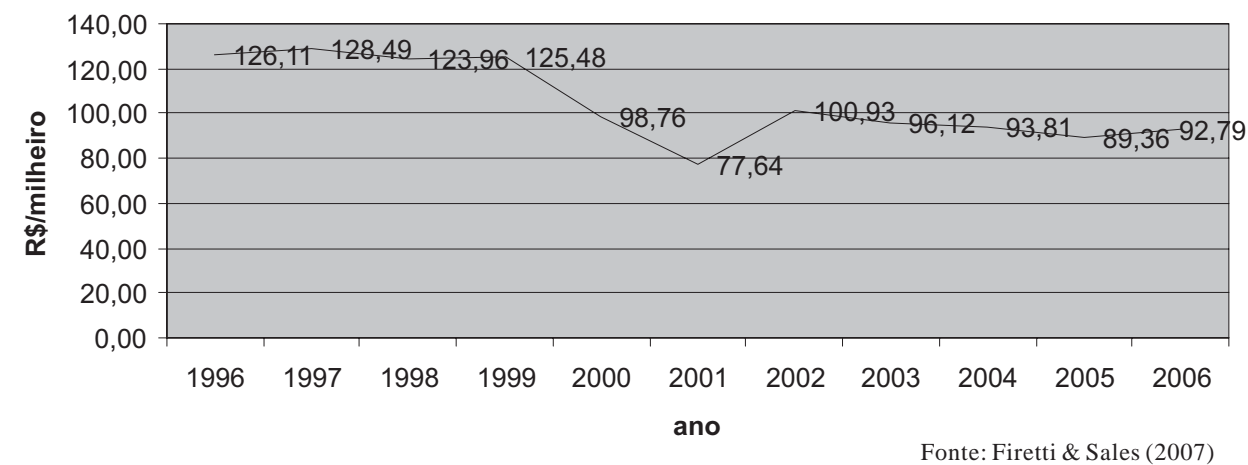

Figura 2 - Preço médio dos alevinos de tilápia praticados por produtores do estado de São Paulo, no período de 1996 a 2006. 


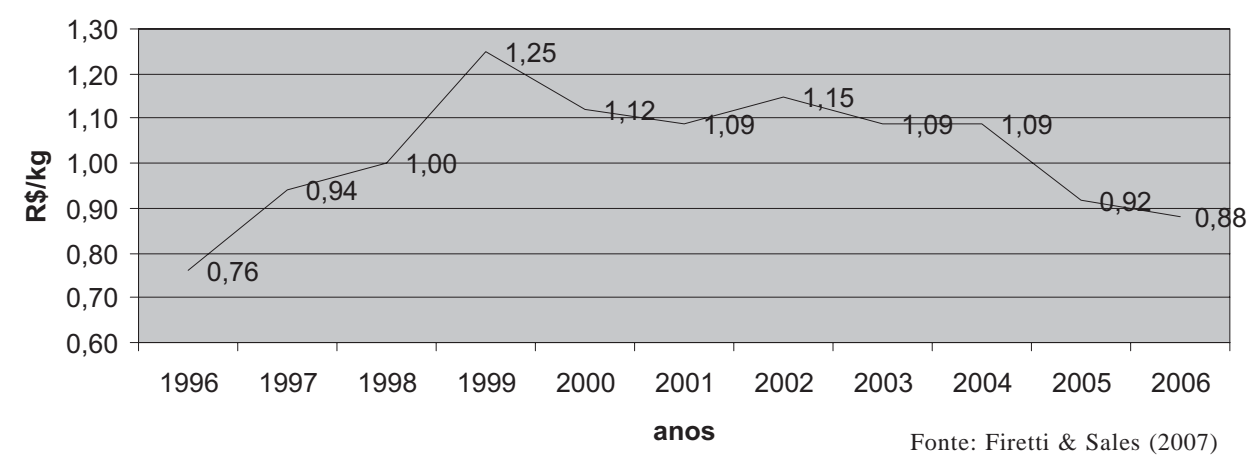

Figura 3 - Preço médio do quilo de ração praticados por produtores do estado de São Paulo, no período de 1996 a 2006.

R\$ 92,76 em 2006. Eles mostram como causa desta desvalorização a maturidade tecnológica do processo produtivo, a competitividade entre fornecedores e o aumento da oferta. Acrescenta-se a estes fatores o menor preço pago pelos pesqueiros particulares (pesque-pagues) e pelos frigoríficos, forçando a diminuição do custo de produção.

A Figura 2 mostra também que, o ano de 1999 foi um divisor de águas para a tilapicultura paulista, Após este ano os preços não voltaram ao patamar de $\mathrm{R} \$ 125,00$ o milheiro conseguido naquele ano.

Ração

Segundo o Sindicato Nacional da Indústria de Alimentação Animal - SINDIRAÇÕES, em 2009, o Brasil produziu 58,4 milhões de toneladas de ração para alimentação animal. Deste total a aquicultura representou 0,65\% (380.000 toneladas). Uma fração muito pequena, principalmente quando comparada à produção de ração para aves que representou 55,9\% (32.640.000 toneladas). Mas quando comparamos o crescimento anual entre 2008 e 2009 esta situação se inverte, uma vez que, a aquicultura cresceu $17 \%$ e a avicultura apenas 1\%. É bom lembrar que os $1 \%$ da produção de ração para aves corresponde a 377.000 toneladas, enquanto $17 \%$ da produção de ração para organismos aquáticos correspondem a 56.000 toneladas. Em 2010, a estimativa do SINDIRAÇÕES, é de produzir 60,4 milhões de toneladas de ração para a alimentação animal, sendo destas 429.000 toneladas de ração para a alimentação de organismos aquáticos, um crescimento estimado de 12,89\% entre 2009 e 2010.

Das 380.000 toneladas de ração produzidas para aquicultura, 78,9\% destina-se a alimentação de peixes (300.000 toneladas) e 21,1\% destina-se a alimentação de camarões marinhos (80.000 toneladas). (Sindirações, 2010).

O insumo ração representa 40 a $60 \%$ do custo total de produção da aquicultura. Valor relativamente alto quando comparado aos demais itens do custo. Esta representatividade nos leva a dar a ração, e ao manejo alimentar a ser adotado, uma atenção especial.

O Brasil tem buscado linhagens de tilápias melhoradas visando melhor desempenho zootécnico, melhor aproveitamento dos alimentos e assim diminuindo o consumo. Também, estão sendo pesquisadas e produzidas melhores rações, isto é, com melhor digestibilidade, pois, rações mais eficientes, mesmo com preços mais altos podem possibilitar um menor custo de produção.

Ainda, temos na ração a grande vilã do custo de organismos aquáticos.

Tendo como referencia o artigo de Firetti \& Sales (2007), a análise do comportamento dos preços da ração ao longo dos anos nos mostra que este insumo tem aumentado ao longo dos anos.

Segundo Firetti \& Sales (2007), o preço do quilo da ração que era de $\mathrm{R} \$ 0,76$ em 1996 passou a $\mathrm{R} \$ 0,88$ em 2006, aumento de 15,81\%. Mais uma vez o ano de 1999 aparece como um marco na série histórica, neste ano, como cita Firetti \& Sales (2007), o preço da ração foi, ainda, mais alto, quando o quilo da ração foi vendido por $\mathrm{R} \$ 1,25$.

Sales et al. (2005) comentam que, desde 2002, a piscicultura nacional passa por um processo de reestruturação, reflexo do recrudescimento da crise econômica e dos problemas no abastecimento de matériasprimas para rações e no escoamento da produção. Informações obtidas na época com piscicultores paulista a crise se iniciou em 1999 quando os pesqueiros particulares diminuíram a demanda, sobrando peixe nas propriedades, e o custo de produção aumentou com o aumento dos preços dos insumos.

Em termos de comparação, em 1996, o preço de venda do quilo da tilápia e o custo operacional efetivo médio, citados por Firetti \& Sales (2007) eram de R \$ 5,25 e R \$2,86, respectivamente. Em 2006, estes valores foram de R 2,73 para o quilo da tilápia pago ao produtor sendo do custo 
operacional efetivo médio de R $\$ 1,74$, : Estes valores fizeram com que a margem bruta média sofresse uma redução de 142,4\% passando de R\$2,40, em 1996 para R \$ 0,99 em 2006.

\section{Considerações Finais}

Os outros insumos, como corretivos, desinfetantes, medicamentos, etc., ainda tem pouca representatividade no custo de produção, segundo Martin et al. (1995) estes componentes representariam média, em torno de $0,7 \%$ do custo de produção de tilápias em vários sistemas de produção em São Paulo.

Podemos inferir a que a atividade de tilapicultura vem passando, desde as décadas passadas, por um processo de profissionalização, com o produtor mais atento ao manejo e aos insumos utilizados. Hoje, já é possível escolher o insumo que mais se adapta ao manejo empregado pelo produtor. A mudança de comportamento de alguns produtores mostra que a escolha do insumo pelo seu preço de venda não é uma prática generalizada. Alguns piscicultores se utilizam de ferramentas informatizadas para acompanhar o desempenho produtivo e econômico de suas criações.

Novos mercados se abrem a cada safra, fazendo com que a tilápia, cada vez mais, ganhe a preferência do consumidor. Também, é notória a concorrência de algumas novas e velhas espécies. As velhas, tais como salmão e merluza continuam a concorrer com a tilápia e a entrada no mercado nacional do filé do peixe vietnamita "panga" (Pangasius hipophthalmus) tem incomodado os produtores de tilápia de várias regiões do pais, a preços muito abaixo do praticado pelas processadoras de tilápia o filé do "panga" tem tido uma procura intensa por parte de consumidores que buscam preços menores sem se preocupar com a qualidade do produto.

\section{Referências}

CAMPOS, C. M.C.; GANECO, L. N.; CASTELLANI, D.; MARTINS, M.I.E.G. 2007. Avaliação econômica da criação de tilápias em tanque-rede, município de Zacarias, SP. Boletim do Instituto de Pesca, v.33, n.2, p.225-261

CARNEIRO, P.C.F.; MARTINS, M.I.E.G.; CYRINO, J.E.P. 1999. Estudo de Caso da Criação comercial da tilápia vermelha em tanques-rede - avaliação econômica Informações Econômicas, SP,v.29,n.8, ago.1999.p- 52-61

CASTAGNOLLI, N. 1996 Aquicultura para o ano 2000. Brasília: CNPq, 1996. 96p.

FAO, 2009. Disponivel em http://www.fao.org/docrep/010/a1495e/ a1495e00.HTM

FIRRETTI, R.; SALES, D.S.; GARCIA, S.M. Lucro com tilápia é para profissionais Anualpec 2007. Anuário da Pecuária Brasileira. São Paulo: Instituto FNP, 2007. p.285-286.

SALES, D.S.; CASEIRO, A.; FIRRETTI, R. et al. O desenvolvimento recente da aquicultura brasileira. Anualpec. Anuário da Pecuária Brasileira. São Paulo: Instituto FNP, 2005. p.252257.

FITZSIMMONS, K. [2010]. Tilapia: 2009 State of the Industry Report. WAS2010, San Diego,CA. Disponível em: https:// w w w. w a s.org/W a s M e e t i n g / M e e t i n g s/ SessionAbstracts.aspx?Code $=A Q 2010 \&$ Session $=31$. Acesso em 27 de junho de 2010.

MARTIN,N.B.; et al. 1995. Custos e Retornos na Piscicultura em Sao Paulo.Informações Econômicas, SP, v.25, n.1, jan. p.939..

SANTOS, V.B. [2006]. A disponibilidade de diferentes linhagens de tilápia. Disponível em: http:// www.apta.sp.gov.br/polos/. Maio de 2006. Acesso em: 29/6/ 2010.

SCORVO-FILHO, MARTIN, N.B. AYROZA, L.M. da S. 1999. Preços na Piscicultura no Estado de São Paulo, 1995-1997. Informações Econômicas, v.29, n.3, p-1-10, 1999.

VALENTI, W.C.; POLI, C.R.; PEREIRA, J.A.; BORGHETTI, J.R. Aquicultura no Brasil: bases para um desenvolvimento sustentável. Brasília: CNPq, 2000. 399p.

ZIMmermann, S.A. [2002]. Tilápia na América Latina Introdução e Situação Atual. In https://www.was.org/LACWAS/boletins/boletim02/02_reportagem/02port_2.htm http:/ /www.fao.org/news/story/es/item/41570/icode/(1) Documento preparado a partir da palestra apresentada no VII SIMPÓSIO BRASILEIRO DE MELHORAMENTO ANIMAL, promovido pela Sociedade Brasileira de Melhoramento Animal e realizado em São Carlos (SP), de 10 a 12 de Julho de 2008 (www.sbma.com.br). 